\title{
Measuring Primary Care Across 35 OECD Countries
}

Stephen J. Zyzanski, $P b D^{1}$

Martha M. Gonzalez ${ }^{2}$

Jonathan P. O'Neal ${ }^{2}$

Rebecca S. Etz, $P b D^{2}$

Sarab R. Reves, FNP-C, MBA ${ }^{2}$

Kurt C. Stange, $M D, P b D^{1,2,3}$

${ }^{1}$ Center for Community Health Integration, Departments of Family Medicine \& Community Health, Population \& Quantitative Health Sciences, Case Western Reserve University, Cleveland, Ohio

${ }^{2}$ Larry A. Green Center for the Advancement of Primary Health Care for the Public Good, Department of Family Medicine and Population Health, Virginia Commonwealth University, Richmond, Virginia

${ }^{3}$ Case Comprehensive Cancer Center, Case Western Reserve University, Cleveland, Ohio

Conflicts of interest: authors report none.

\section{CORRESPONDING AUTHOR}

Rebecca S. Etz

VCU Department of Family Medicine and Population Health

One Capital Square Building 830 East Main Street, Rm 629

Richmond, VA 23298-0101

Rebecca.Etz@vcuhealth.org

\begin{abstract}
PURPOSE To examine the psychometric properties and scores of the PersonCentered Primary Care Measure (PCPCM) in 28 languages and 35 Organisation for Economic Co-operation and Development (OECD) countries.
\end{abstract}

METHODS Using a paid online sampling service, we requested age- and sex-representative samples of 360 adults in each country. We administered the PersonCentered Primary Care Measure-a previously validated 11-item, patient-reported measure that was developed using what patients and clinicians said is most important about primary care. We also assessed construct validity through associations with demographics, the Patient-Enablement Instrument, number of years the person had been with their primary care physician and practice, whether the patient thought the doctor knowing the results would improve their care, and whether it was hard to complete the survey. We assessed the psychometric properties of the PCPCM in each country and report the summative and item-specific PCPCM scores for each country.

RESULTS The PCPCM exhibited solid psychometric properties across all languages and countries, with Cronbach's alphas ranging from 0.88 to 0.95 , and corrected item-total correlations ranging from 0.47 to 0.81 , with the vast majority of countries ranging from the low $0.50 \mathrm{~s}$ to the high $0.70 \mathrm{~s}$. Multiple analyses showed strong evidence of concurrent validity. With a potential range from a low of 1 to a high of 4 , the overall mean score was 2.74 , with a standard deviation of 0.19 . Mean PCPCM scores ranged from the lowest in Sweden (2.28) to the highest in Turkey (3.08), with Germany ranking second (3.01), and the United States third (2.99).

CONCLUSIONS The internal consistency and concurrent validity of the PCPCM across multiple countries provides strong evidence of the coherence of the breadth of primary care functions that patients and clinicians say are important. The diversity of total and item-specific scores across countries provokes interesting hypotheses about the influence of each different country's policies, practices, demographics, and culture on primary care, and provides a strong impetus for further ecological and individual data analyses using the Person-Centered Primary Care Measure.

Ann Fam Med 2021;19:Online. https://doi.org/10.1370/afm.2697.

Annals "Online First" article. Accepted for publication in a later issue.

\section{INTRODUCTION}

$\mathrm{P}$ rimary care is widely viewed as the cornerstone of a high-functioning health care system. ${ }^{1-4}$ Considerable variability in health care system design and in how primary care is provided presents an opportunity to learn from different approaches, ${ }^{5}$ in order to better understand and improve what matters about primary care. ${ }^{6}$ Most system-level measures of primary care, however, are crude in measuring what actually happens regarding the multiple functions of primary care. With a few limited exceptions, including the Primary Care Assessment Tool, ${ }^{7}$ World Health Organization's (WHO) Primary Care Evaluation Tool (PCET), ${ }_{1}^{8,9}$ and measures developed for the Quality and Costs of Primary Care in Europe (QUALICOPC) study, ${ }_{1}^{10}$ most patient-report measures that are sensitive to the actual delivery of personalized care have not been translated or validated for use in multiple countries. ${ }^{11,12}$ 
As published previously in this journal ${ }_{1}^{13}$ the Person-Centered Primary Care Measure (PCPCM) is an 11-item, patient-reported measure that assesses the broad scope and integrative, comprehensive nature of primary care including: accessibility, advocacy, community context, comprehensiveness, continuity, coordination, family context, goal-oriented care, health promotion, integration, and relationship. Developed with extensive prior research, the PCPCM is based on what hundreds of patients, clinicians, and to a lesser extent payers say matters in primary care. ${ }^{14}$ The breadth of characteristics included in the measure reflect the complex role of a personal physician and a primary care practice. The PCPCM has been validated in 3 large Internet samples and 11 smaller clinical samples. ${ }^{13}$ We translated the PCPCM from American English into the 27 other primary languages used in Organisation for Economic Co-operation and Development (OECD) countries. ${ }^{15}$

The purpose of this study is to examine the reliability and validity of the Person-Centered Primary Care Measure in 28 languages and 35 countries, and to explore differences in primary care across 35 OECD Countries. We aim to make the PCPCM available for use across diverse languages and countries, and to stimulate preliminary hypotheses about inter-country differences that can be examined in future research to guide policy and practice based on the natural experiment of different countries approaches to health care.

\section{METHODS}

We engaged a company with considerable global and corporate experience (Morningside Translations LLC) to translate the PCPCM from English (USA) into 27 languages used as primary languages in OECD countries. (The surveys fielded in Spain were in [European] Spanish, and the ones in Chile and Mexico were in Latin American Spanish. The UK surveys were in British English.) Items and instructions were translated and then back-translated, with attention to cultural as well as linguistic distinctions, and then reviewed by the study team. In a few instances, we engaged additional input from members of relevant cultural and linguistic groups to resolve any questions about item appropriateness. The translated PCPCMs are available for public use, along with implementation and scoring guidance, on the website of the Larry A. Green Center for the Advancement of Primary Health Care for the Public Good. ${ }^{15}$

We contracted with SurveyMonkey (SurveyMonkey Inc) to reach a sample of 360 survey respondents representing the adult population demographics in each of the 36 OECD countries in 2019.
The survey captured population demographics (male, female, trans, other), age (aged $<18,18-29,30-44$, $45-60,>60$ years), and whether respondents considered themselves to be a member of a minority, but we were not able to specifically sample on these characteristics.

Success of the recruitment strategy was determined by the panel size of SurveyMonkey's respondents within the given country. Because there were no screener questions to determine whether a respondent fit the criteria to complete the survey, SurveyMonkey sent the survey to as many respondents as necessary until the minimum number of responses were collected (in this case, 360). Those that received the survey in their country were given the option of completing the survey or skipping it. Once enough respondents completed the survey, SurveyMonkey stopped sending the survey to more potential respondents.

For Iceland, SurveyMonkey was unable to provide sufficient sample size, and that country was excluded. For Luxembourg, SurveyMonkey was able to obtain a sample size sufficient for validation $(n=150)$, but not the 360 requested that would allow for future analyses. A sample size of 360 allows sufficient power to detect small effect sizes in statistical analyses ${ }_{1}^{16}$ but for this ecological study, the statistics involved only descriptive and psychometric analyses.

In these samples, we fielded an online survey translated to the predominant language in each OECD country. For Belgium, where residents are nearly equally split in their primary use of 2 languages, the survey was preceded by an introductory question asking whether respondents would prefer to take the survey in Dutch or French (49.9\% chose Dutch and 50.1\% chose French). The survey consisted of the PCPCM and questions about demographics, the PatientEnablement Instrument (PEI) ${ }^{17-20}$ number of years the person had been with their primary care physician and practice, and whether the patient thought the doctor knowing the results would improve their care.

In order to have confidence in interpreting each country's PCPCM score, especially given multiple language translations and cultures, it was necessary to reestablish the psychometric properties of the PCPCM for each country. This analysis consisted of providing country-specific evidence of the measure's reliability and concurrent validity. Evidence of the measure's internal consistency reliability was provided by examining the item-total correlations and by computation of Cronbach's $\alpha$ reliability coefficient. Evidence in support of the measure's concurrent validity was provided by examining the correlation of the PCPCM with the PEI and by examining the associations of PCPCM scale scores with important single item outcomes, such as years with the current physician and practice and by 
asking if knowing the results would help the physician understand the respondent's feeling about their health care. Taken together, these analyses are designed to provide evidence that the PCPCM item set was working effectively in each country, as documented by their psychometric properties, and thereby increasing confidence in the interpretation of the country-specific PCPCM scores.

We also report the total and item-specific scores for each country and include the $P$ value for Bonferroni adjustment for multiple hypothesis testing.

This study protocol was reviewed by Virginia Commonwealth University's Institutional Review Board.

\section{RESULTS}

The 35 mean PCPCM scale scores exhibited a wide range of scores from Sweden (lowest observed score: 2.28) to Turkey (highest observed score: 3.08 ). The overall mean of the 35 countries was 2.74 with a standard deviation of 0.19 . Given this range and the small standard deviation, it is clear that there are many inter-country differences in PCPCM mean scores (Table 1). Since each item represents a separate and important domain of primary care, comparative item analyses among countries can be informative (Table 2). Moreover, examining the item means in relation to each country's total score helps to understand which domains contributed the most to the total score.

Examining the instrument's psychometric properties, the 11 item-total correlations for each country exhibited a solid range of correlations ranging from 0.47 to 0.81 with the vast majority of countries ranging from the low 0.50 s to the high $0.70 \mathrm{~s}$. Not one item in any of the 35 countries exhibited an inconsistent association with the total score. Consistent with these ranges are the country-specific Cronbach $\alpha$ reliabilities. Alpha reliabilities ranged from 0.88 to 0.95 . These high reliability coefficients indicate a high degree of internally consistent responses by the respondents. Taken together, these reliability analyses indicate the 11 -item scale was performing as designed despite language translations and cultural differences.

Evidence of concurrent validity is provided by the solid correlations of PCPCM scores and scores on the PEI (Table 1). These Pearson correlations ranged from 0.57 to 0.74 and provide initial evidence of concurrent validity.

Further evidence of concurrent validity is provided by the association of PCPCM scores with singleitem outcomes. Substantial rank-ordered associations were observed between mean PCPCM scores and responses to the item: "How many years have you known this doctor?" Twenty-seven countries (77\%) had a significant association with this item outcome. The results consistently showed, the more years with the doctor, the higher the PCPCM score.

Similarly, strong associations were observed with the item: "How many years have you been a patient at this practice?" Nineteen countries (54\%) had a significant association with this item outcome. In general, the more years with the practice, the higher the PCPCM score.

Next, consistent and strong associations were observed between PCPCM scores and the item: "If your doctor or practice received the answers to these questions, would it help them to understand how you feel about your care?" In 33 of the 35 countries (94\%), the respondents that said yes to this question had substantially higher PCPCM scores than those that responded with a no.

Finally, several associations were observed with the variables of age and sex. Eight countries (23\%) demonstrated an association between patient age and PCPCM scores. In the majority of instances, these associations were not rank ordered. Often both younger and older patients exhibited higher scores while in other associations, middle-age patients scored higher. Sex differences were observed in 8 countries (23\%) with the majority of associations indicating males scoring higher than females.

A final analysis examined PCPCM scores associated with the item: "Was it hard to complete this form?" Only 2 countries (6\%) showed an association of PCPCM scores with this variable, Japan and Chile, but in opposite directions. In Japan respondents who answered no to this question had higher PCPCM scores than those that said yes. In Chile, the opposite was true, those that responded yes had higher scores than those reporting no.

\section{DISCUSSION}

Taken together, the strong and generally consistent psychometric results support the use of the PCPCM in each respective country. The reliability results were solid across all countries and the concurrent validity findings were likewise solid given the associations with the PEI and the single item outcomes.

This study builds on prior research using somewhat different measures to compare countries and to assess the role of primary care in achieving effective and universal coverage, ${ }^{21}$ and provides a basis for further comparative research. ${ }^{10,22-24}$ The grounding of the PCPCM in careful analysis of what patients, clinicians, and employers say is important in primary care $^{13}$ and provides a particularly strong basis for further research. The consistency with which the broad constructs assessed by the PCPCM are understood 
Table 1. Psychometric Properties and Evidence of Concurrent Validity for the PCPCM in 35 Countries

\begin{tabular}{|c|c|c|c|c|c|c|c|c|c|c|c|}
\hline Country & Language & $\begin{array}{c}\text { Mean } \\
\text { (SD) }\end{array}$ & $\alpha$ & $\begin{array}{l}\text { Item- } \\
\text { Total } \\
\text { Corr }\end{array}$ & $\begin{array}{l}\text { Years } \\
\text { MD }\end{array}$ & $\begin{array}{l}\text { Years } \\
\text { With } \\
\text { Practice }\end{array}$ & $\begin{array}{c}\text { MD } \\
\text { Results }\end{array}$ & $\begin{array}{l}\text { PEI } \\
\text { Corr }\end{array}$ & Age & Sex ${ }^{a}$ & Form \\
\hline Turkey & Turkish & $3.08(0.7)$ & 0.95 & $0.70-0.81$ & $0.0001^{b}$ & 0.034 & 0.0001 & 0.74 & NS & $\mathrm{F}$ & NS \\
\hline Germany & German & $3.01(0.62)$ & 0.9 & $0.57-0.71$ & 0.003 & 0.004 & 0.001 & 0.64 & NS & NS & NS \\
\hline United States & English & $2.99(0.77)$ & 0.94 & $0.66-0.81$ & 0.0001 & 0.0001 & 0.0001 & 0.69 & NS & NS & NS \\
\hline Belgium & $\begin{array}{l}\text { Dutch or } \\
\text { French }\end{array}$ & $2.98(0.63)$ & 0.9 & $0.53-0.72$ & NS & NS & 0.0001 & 0.57 & NS & NS & NS \\
\hline France & French & $2.96(0.69)$ & 0.91 & $0.57-0.74$ & 0.008 & 0.014 & 0.0001 & 0.62 & 0.047 & NS & NS \\
\hline Luxembourg & Luxembourgish & $2.92(0.64)$ & 0.88 & $0.55-0.65$ & NS & NS & NS & 0.67 & NS & NS & NS \\
\hline Austria & Austrian & $2.92(0.64)$ & 0.9 & $0.52-0.78$ & 0.0001 & 0.0001 & 0.0001 & 0.69 & NS & M & NS \\
\hline Australia & English & $2.9(0.7)$ & 0.93 & $0.61-0.79$ & 0.0001 & 0.01 & 0.0001 & 0.7 & NS & NS & NS \\
\hline Switzerland & German & $2.87(0.63)$ & 0.9 & $0.50-0.69$ & NS & 0.01 & 0.0001 & 0.62 & NS & NS & NS \\
\hline Spain & Spanish & $2.87(0.65)$ & 0.92 & $0.61-0.78$ & 0.0001 & NS & 0.0001 & 0.74 & NS & NS & NS \\
\hline Hungary & Hungarian & $2.87(0.72)$ & 0.92 & $0.47-0.80$ & 0.009 & NS & 0.0001 & 0.63 & NS & NS & NS \\
\hline Norway & Norwegian & $2.85(0.68)$ & 0.92 & $0.64-0.74$ & 0.008 & 0.009 & 0.0001 & 0.67 & NS & NS & NS \\
\hline New Zealand & English & $2.82(0.7)$ & 0.92 & $0.56-0.77$ & 0.001 & 0.004 & 0.0001 & 0.62 & NS & NS & NS \\
\hline Ireland & English & $2.82(0.69)$ & 0.91 & $0.57-0.74$ & 0.005 & NS & 0.0001 & 0.68 & NS & NS & NS \\
\hline Portugal & Portuguese & $2.8(0.63)$ & 0.91 & $0.57-0.77$ & 0.0001 & 0.021 & 0.003 & 0.67 & NS & M & NS \\
\hline Mexico & Spanish & $2.78(0.73)$ & 0.92 & $0.57-0.78$ & 0.0001 & 0.0001 & 0.0001 & 0.68 & NS & NS & NS \\
\hline Slovenia & Slovenian & $2.77(0.72)$ & 0.93 & $0.61-0.76$ & 0.0001 & NS & 0.0001 & 0.69 & 0.039 & NS & NS \\
\hline Italy & Italian & $2.77(0.77)$ & 0.94 & $0.54-0.82$ & 0.001 & 0.0001 & 0.0001 & 0.73 & NS & M & NS \\
\hline Denmark & Danish & $2.77(0.69)$ & 0.91 & $0.52-0.77$ & 0.0001 & 0.0001 & 0.0001 & 0.61 & NS & NS & NS \\
\hline Latvia & Latvian & $2.76(0.7)$ & 0.91 & $0.58-0.73$ & NS & NS & 0.0001 & 0.63 & NS & NS & NS \\
\hline Netherlands & Dutch & $2.75(0.69)$ & 0.92 & $0.52-0.76$ & NS & NS & 0.0001 & 0.59 & NS & M & NS \\
\hline Canada & English & $2.75(0.8)$ & 0.95 & $0.69-0.83$ & 0.0001 & 0.0001 & 0.0001 & 0.72 & NS & NS & NS \\
\hline Slovakia & Slovak & $2.71(0.69)$ & 0.91 & $0.54-0.73$ & 0.004 & 0.005 & 0.0001 & 0.71 & NS & NS & NS \\
\hline Estonia & Estonian & $2.69(0.66)$ & 0.9 & $0.53-0.77$ & NS & NS & 0.0001 & 0.57 & 0.03 & NS & NS \\
\hline Czech & Czech & $2.69(0.64)$ & 0.9 & $0.51-0.71$ & NS & NS & 0.0001 & 0.68 & NS & NS & NS \\
\hline $\begin{array}{l}\text { United } \\
\text { Kingdom }\end{array}$ & English & $2.61(0.73)$ & 0.92 & $0.59-0.80$ & 0.0001 & NS & 0.0001 & 0.71 & NS & M & NS \\
\hline Israel & Hebrew & $2.61(0.66)$ & 0.89 & $0.48-0.73$ & 0.039 & NS & 0.0001 & 0.63 & NS & NS & NS \\
\hline Finland & Finnish & $2.56(0.61)$ & 0.91 & $0.57-0.73$ & 0.0001 & 0.003 & 0.001 & 0.68 & 0.001 & NS & NS \\
\hline Greece & Greek & $2.54(0.63)$ & 0.93 & $0.66-0.76$ & 0.035 & NS & 0.005 & 0.67 & 0.002 & NS & NS \\
\hline Poland & Polish & $2.52(0.67)$ & 0.9 & $0.48-0.75$ & 0.0001 & NS & 0.0001 & 0.68 & NS & NS & NS \\
\hline Korea & Korean & $2.5(0.67)$ & 0.92 & $0.60-0.75$ & NS & NS & 0.0001 & 0.67 & 0.013 & NS & NS \\
\hline Lithuania & Lithuanian & $2.47(0.66)$ & 0.89 & $0.47-0.73$ & 0.0001 & NS & 0.0001 & 0.63 & NS & NS & NS \\
\hline Japan & Japanese & $2.46(0.67)$ & 0.94 & $0.69-0.84$ & 0.0001 & 0.0001 & 0.0001 & 0.73 & NS & NS & 0.0001 \\
\hline Chile & Spanish & $2.41(0.76)$ & 0.92 & $0.56-0.77$ & 0.0001 & 0.002 & NS & 0.73 & 0.02 & M & 0.04 \\
\hline Sweden & Swedish & $2.28(0.74)$ & 0.93 & $0.67-0.80$ & 0.0001 & 0.0001 & 0.0001 & 0.74 & 0.034 & M & NS \\
\hline \multicolumn{12}{|c|}{$\begin{array}{l}\text { Corr = correlation; Form = PCPCM correlation with whether the patient reported it was hard to complete the survey; MD Results = PCPCM correlation with patient } \\
\text { report of whether the doctor knowing the results would improve their care; PEl-Cor = PCPCM correlation with score on the Patient Enablement Index; PCPCM = Per- } \\
\text { son-Centered Primary Care Measure; Yrs MD = PCPCM correlation with number of years the patient had been with the physician; Yrs Pract = PCPCM correlation with } \\
\text { number of years the patient had been with the practice. }\end{array}$} \\
\hline \multicolumn{12}{|c|}{ Note: The Spanish and Portuguese translations were used with either European or Latin American versions, as appropriate to the country. } \\
\hline Significant sex & rences, $P$ values & from Turke & .000 & Austria (0 & & 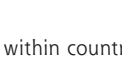 & & & & & \\
\hline
\end{tabular}

and appreciated by patients/the public across 35 countries with different cultures and health systems provides evidence of the universal internal consistency and conceptual coherence of the breadth of primary care attributes.

The variability of the total score and individual items across countries is interesting. We encourage readers with local knowledge of each country to use this journal's e-letter feature to share hypotheses that might explain these differences.

At the extremes-the high scores for Turkey may reflect reforms of the past decade that have attempted to increase the availability of primary care, and previous high levels of patient satisfaction with their 
Table 2. Person-Centered Primary Care Item Scores by Country (Ranked by Total Score)

\begin{tabular}{|c|c|c|c|c|c|c|c|c|c|c|c|c|}
\hline Country & Total & Q1 & Q2 & Q3 & Q4 & Q5 & Q6 & Q7 & Q8 & Q9 & Q10 & Q11 \\
\hline Turkey & 3.08 & 3.3 & 3.2 & 3.2 & 2.9 & 2.8 & 2.9 & 3.1 & 3.0 & 3.1 & 3.2 & 3.1 \\
\hline Germany & 3.01 & 3.2 & 3.1 & 3.2 & 3.0 & 3.3 & 2.6 & 3.0 & 2.8 & 3.0 & 3.0 & 2.9 \\
\hline United States & 2.99 & 3.1 & 3.1 & 3.2 & 3.0 & 3.0 & 2.5 & 2.8 & 2.9 & 2.9 & 3.2 & 3.0 \\
\hline Belgium & 2.98 & 3.1 & 3.0 & 3.3 & 2.9 & 3.2 & 2.6 & 3.0 & 3.0 & 2.8 & 3.1 & 2.9 \\
\hline France & 2.96 & 3.2 & 3.0 & 3.3 & 3.0 & 3.2 & 2.5 & 2.8 & 2.9 & 2.5 & 3.2 & 2.9 \\
\hline Austria & 2.92 & 3.3 & 3.1 & 3.2 & 3.0 & 3.2 & 2.4 & 2.9 & 2.6 & 2.7 & 3.0 & 2.7 \\
\hline Luxembourg & 2.92 & 3.3 & 3.0 & 3.1 & 2.9 & 3.0 & 2.5 & 2.9 & 2.7 & 2.7 & 3.1 & 2.8 \\
\hline Australia & 2.90 & 3.1 & 3.1 & 3.3 & 3.0 & 3.0 & 2.5 & 2.8 & 2.8 & 2.7 & 3.0 & 2.8 \\
\hline Spain & 2.87 & 3.2 & 3.0 & 3.3 & 3.0 & 2.9 & 2.2 & 2.8 & 2.7 & 2.7 & 3.0 & 2.8 \\
\hline Hungary & 2.87 & 3.1 & 2.8 & 3.2 & 3.0 & 3.0 & 2.8 & 2.8 & 2.8 & 2.4 & 3.0 & 2.6 \\
\hline Switzerland & 2.87 & 3.1 & 3.0 & 3.1 & 3.0 & 3.0 & 2.4 & 2.9 & 2.7 & 2.7 & 2.9 & 2.8 \\
\hline Norway & 2.85 & 3.0 & 3.0 & 3.0 & 2.8 & 2.9 & 2.6 & 2.9 & 2.7 & 2.7 & 2.9 & 2.8 \\
\hline Ireland & 2.82 & 3.1 & 3.0 & 3.2 & 2.7 & 2.9 & 2.4 & 2.8 & 2.8 & 2.5 & 2.9 & 2.7 \\
\hline New Zealand & 2.82 & 3.0 & 3.1 & 3.2 & 2.9 & 2.8 & 2.3 & 2.8 & 2.7 & 2.5 & 2.9 & 2.7 \\
\hline Portugal & 2.80 & 3.1 & 3.0 & 3.2 & 2.9 & 2.9 & 2.1 & 2.8 & 2.6 & 2.4 & 3.0 & 2.8 \\
\hline Mexico & 2.78 & 3.1 & 3.1 & 3.2 & 2.7 & 2.8 & 2.2 & 2.5 & 2.7 & 2.5 & 3.0 & 2.7 \\
\hline Denmark & 2.77 & 3.0 & 2.9 & 3.1 & 2.8 & 2.6 & 2.9 & 2.8 & 2.6 & 2.5 & 2.7 & 2.8 \\
\hline Slovenia & 2.77 & 2.9 & 2.8 & 3.1 & 2.8 & 2.9 & 2.3 & 2.9 & 2.7 & 2.6 & 2.9 & 2.6 \\
\hline Italy & 2.77 & 3.0 & 2.8 & 3.0 & 2.6 & 3.0 & 2.2 & 2.7 & 2.8 & 2.8 & 2.9 & 2.7 \\
\hline Latvia & 2.76 & 3.0 & 2.7 & 3.0 & 2.9 & 2.8 & 2.6 & 2.6 & 2.7 & 2.5 & 2.9 & 2.6 \\
\hline Canada & 2.75 & 2.9 & 2.9 & 3.0 & 2.7 & 2.8 & 2.4 & 2.7 & 2.7 & 2.5 & 2.9 & 2.7 \\
\hline Netherlands & 2.75 & 2.9 & 2.8 & 3.0 & 2.7 & 2.8 & 2.2 & 2.8 & 2.8 & 2.7 & 2.8 & 2.7 \\
\hline Slovak & 2.71 & 3.1 & 3.0 & 3.1 & 2.8 & 2.9 & 2.2 & 2.8 & 2.6 & 2.0 & 2.8 & 2.5 \\
\hline Estonia & 2.69 & 3.0 & 2.9 & 3.1 & 2.9 & 2.6 & 2.4 & 2.7 & 2.5 & 2.5 & 2.7 & 2.4 \\
\hline Czech & 2.69 & 3.2 & 3.0 & 3.2 & 2.8 & 2.7 & 1.8 & 2.8 & 2.6 & 2.4 & 2.8 & 2.3 \\
\hline United Kingdom & 2.61 & 2.9 & 3.0 & 3.0 & 2.8 & 2.5 & 2.1 & 2.5 & 2.4 & 2.4 & 2.7 & 2.5 \\
\hline Israel & 2.61 & 2.8 & 2.8 & 3.1 & 2.5 & 2.7 & 2.1 & 2.8 & 2.5 & 2.0 & 2.8 & 2.6 \\
\hline Finland & 2.56 & 3.0 & 3.0 & 2.9 & 2.7 & 2.3 & 1.7 & 2.7 & 2.3 & 2.1 & 2.8 & 2.7 \\
\hline Greece & 2.54 & 2.6 & 2.6 & 2.9 & 2.5 & 2.6 & 2.4 & 2.5 & 2.6 & 2.2 & 2.7 & 2.5 \\
\hline Poland & 2.52 & 2.9 & 2.7 & 2.9 & 2.6 & 2.8 & 2.1 & 2.4 & 2.2 & 2.2 & 2.5 & 2.3 \\
\hline Korea & 2.50 & 3.0 & 2.9 & 2.8 & 2.6 & 2.1 & 2.0 & 2.3 & 2.4 & 2.3 & 2.6 & 2.5 \\
\hline Lithuania & 2.47 & 2.9 & 2.7 & 2.9 & 2.6 & 2.5 & 1.8 & 2.5 & 2.2 & 2.1 & 2.7 & 2.3 \\
\hline Japan & 2.46 & 2.7 & 2.7 & 2.6 & 2.3 & 2.4 & 2.1 & 2.6 & 2.3 & 2.4 & 2.5 & 2.5 \\
\hline Chile & 2.41 & 2.7 & 2.6 & 2.9 & 2.5 & 2.2 & 1.7 & 2.1 & 2.3 & 2.3 & 2.7 & 2.4 \\
\hline Sweden & 2.28 & 2.8 & 2.7 & 2.6 & 2.5 & 2.0 & 1.8 & 2.1 & 1.8 & 2.0 & 2.4 & 2.3 \\
\hline
\end{tabular}

treatment by family doctors. ${ }^{25}$ The low scores for Sweden may surprise some who have heard of the strong Swedish welfare state and relatively homogeneous culture. However, despite many health care system and primary care strengths and reforms, ${ }_{1}^{26}$ according to one analyst: "In Sweden, and increasingly even in traditional strongholds of general practice, team-based primary care is thought to respond to increasing demands, filtering out non- and minor disease through triage, practicing task distribution, and moving the GP to a secondary level working with the 'really sick', in all a decline in direct contact between patient and GP. Conclusions: When this happens, clinical medicine as a whole becomes drained of the practice of its human dimension." ${ }^{27}$

We wonder if the relatively high scores for the United States (ranked \#3), given inter-country rankings using different measures that rate US health care and primary care more poorly, ${ }^{28}$ reflects the fact that the PCPCM was developed based largely from data on what US patients, clinicians, and payers say is important, but we invite readers to posit other hypotheses. 
The study is limited by a sampling scheme that, while similar across countries, may not have yielded a representative sample of patients in each country. It also is possible that, despite efforts at linguistically and culturally sensitive translation, some differences between countries could be due to contextual factors affecting interpretation of specific PCPCM items.

Nevertheless, the solid reliability and validity of the PCPCM across different languages and countries, and the variability of responses across countries call for further ecological and individual research using this measure to learn from the natural experiment of different approaches to health care across different countries. ${ }^{29-31}$

\section{To read or post commentaries in response to this article, go to} the e-letters tab at https://doi.org/10.1370/afm.2697.

Key words: primary care; quantitative methods: health status/QOL measurement; measure development; international comparison

Submitted Janurary 22, 2020; submitted, revised, November 14, 2020; accepted January 22, 2021.

Funding support: American Board of Family Medicine Foundation support of the Larry A. Green Center for the Advancement of Primary Health Care for the Public Good, the Dorothy Jones Weatherhead Professorship at Case Western Reserve University, and the University Suburban Health Center.

\section{References}

1. Hansen J, Groenewegen PP, Boerma WG, Kringos DS. Living in a country with a strong primary care system is beneficial to people with chronic conditions. Health Aff (Millwood). 2015;34(9):1531-1537.

2. Kringos DS. Primary care. In: Cockerham WC, Dingwall R, Quah SR, eds. The Wiley Blackwell Encyclopedia of Health, Illness, Behavior, and Society. Vol IV, Me-R. Wiley Blackwell/John Wiley \& Sons, Ltd; 2014: 1875-1879.

3. Donaldson MS, Yordy KD, Lohr KN, Vanselow NA, eds. Primary Care: America's Health in a New Era. National Academy Press; 1996.

4. Starfield B, Shi L, Macinko J. Contribution of primary care to health systems and health. Milbank Q. 2005;83(3):457-502.

5. Meads G. Primary Care in the Twenty-First Century. Radcliffe; 2006.

6. Starfield B. Primary Care: Balancing Health Needs, Services, and Technology. Oxford University Press; 1998.

7. The Johns Hopkins Primary Care Policy Center. Primary care assessment tools. Johns Hopkins Bloomberg School of Public Health. Accessed Jul 31, 2020. https://www.jhsph.edu/research/centers-andinstitutes/johns-hopkins-primary-care-policy-center/pca_tools.html

8. Fracolli LA, Gomes MF, Nabão FR, Santos MS, Cappellini VK, de Almeida AC. Primary health care assessment tools: a literature review and metasynthesis. Cien Saude Colet. 2014;19(12):4851-4860.

9. WHO Commission on Social Determinants of Health. Primary Care Evaluation Tool (PCET). Accessed Aug 8, 2020. https://www. euro.who.int/en/health-topics/Health-systems/primary-health-care/ publications/2010/primary-care-evaluation-tool-pcet

10. Schäfer WL, Boerma WG, Kringos DS, et al. Measures of quality, costs and equity in primary health care instruments developed to analyse and compare primary care in 35 countries. Qual Prim Care. 2013;21(2):67-79.

11. Stange KC, Etz RS, Gullett $H$, et al. Metrics for assessing improvements in primary health care. Annu Rev Public Health. 2014;35: 423-442.
12. Haggerty JL, Lévesque JF, Santor DA, et al. Accessibility from the patient perspective: comparison of primary healthcare evaluation instruments. Healthc Policy. 2011;7(Spec Issue):94-107.

13. Etz RS, Zyzanski SJ, Gonzalez MM, Reves SR, O’Neal JP, Stange KC. A new comprehensive measure of high-value aspects of primary care. Ann Fam Med. 2019;17(3):221-230.

14. Etz RS, Stange KC. Synthesis of the Starfield Summit III: meaningful measures for primary care. Published 2018. Accessed Jul 27, 2020. https://static1.squarespace.com/static/56bb9997746fb9d2b5c7 0970/t/5ab1731a03ce64e295a79b94/1521578778331/StarfieldIII SynthesisRev.pdf

15. Larry A. Green MD Center for the Advancement of Primary Health Care for the Public Good. Measures. Accessed Aug 1, 2020. https:// www.green-center.org/pcpcm

16. Cohen J. Statistical Power Analysis for the Behavioral Sciences. 2nd ed. L. Erlbaum Associates; 1988.

17. Mercer SW, Watt GC. The inverse care law: clinical primary care encounters in deprived and affluent areas of Scotland. Ann Fam Med. 2007;5(6):503-510.

18. Howie JG, Heaney DJ, Maxwell M, Walker JJ, Freeman GK, Rai H. Quality at general practice consultations: cross sectional survey. BMJ. 1999;319(7212):738-743.

19. Howie JG, Heaney DJ, Maxwell M, Walker JJ. A comparison of a Patient Enablement Instrument (PEI) against two established satisfaction scales as an outcome measure of primary care consultations. Fam Pract. 1998;15(2):165-171.

20. Howie JG, Heaney DJ, Maxwell M. Measuring quality in general practice. Pilot study of a needs, process and outcome measure. Occas Pap R Coll Gen Pract. 1997;(75):i-xii, 1-32.

21. van Weel C, Kidd MR. Why strengthening primary health care is essential to achieving universal health coverage. CMAJ. 2018;190(15): E463-E466.

22. Boerma W. Final Report Summary - QUALICOPC (Quality and Costs of Primary Care in Europe). CORDIS/European Commission; 2015. 157428.

23. Schäfer WLA, Boerma WGW, Kringos DS, et al. QUALICOPC, a multicountry study evaluating quality, costs and equity in primary care. BMC Fam Pract. 2011;12(1):115.

24. PHCPI. Measuring primary health care performance. Accessed December 3, 2020. https://improvingphc.org/

25. Kringos DS, Boerma WG, Spaan E, Pellny M. A snapshot of the organization and provision of primary care in Turkey. BMC Health Serv Res. 2011;11:90.

26. Hasvold T. Building primary care in a changing Europe: case studies. In: Kringos DS, Boerma WGW, Hutchinson A, et a, eds. Observatory Studies Series, No. 40. European Observatory on Health Systems and Policies; 2015.

27. Rudebeck CE. Relationship based care - how general practice developed and why it is undermined within contemporary healthcare systems. Scand J Prim Health Care. 2019;37(3):335-344.

28. Commonwealth Fund. Health care system performance rankings. Accessed Dec 3, 2020. https://www.commonwealthfund.org/chart/ 2017/health-care-system-performance-rankings

29. Organization for Economic Co-Operation and Development. OECD health data - frequently requested data. Published 2020. Accessed Jul 31, 2020. https://www.oecd.org/health/health-statistics.htm

30. Macinko J, Starfield B, Shi L. The contribution of primary care systems to health outcomes within Organization for Economic Cooperation and Development (OECD) countries, 1970-1998. Health Serv Res. 2003;38(3):831-865.

31. OECD data. Health spending. Accessed May 30, 2020. https://data. oecd.org/healthres/health-spending.htm 